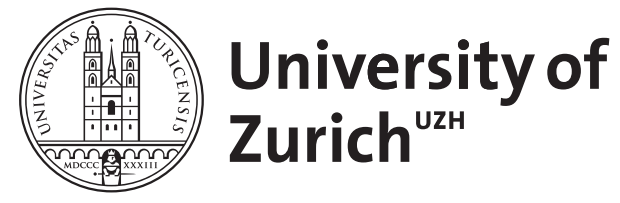

\title{
Hepatic failure due to hepatitis $B$ reactivation in a patient with ulcerative colitis treated with prednisone
}

\author{
Zeitz, J ; Mullhaupt, B ; Fruehauf, H ; Rogler, G ; Vavricka, S R
}

DOI: https://doi.org/10.1002/hep.23035

Posted at the Zurich Open Repository and Archive, University of Zurich ZORA URL: https://doi.org/10.5167/uzh-32056

Journal Article

Originally published at:

Zeitz, J; Mullhaupt, B; Fruehauf, H; Rogler, G; Vavricka, S R (2009). Hepatic failure due to hepatitis B reactivation in a patient with ulcerative colitis treated with prednisone. Hepatology, 50(2):653-654.

DOI: https://doi.org/10.1002/hep.23035 


\section{Hepatic Failure due to Hepatitis B Reactivation in a Patient with}

\section{Ulcerative Colitis Treated with Prednisone}

Jonas Zeitz, Beat Muellhaupt, Heiko Fruehauf, Gerhard Rogler, Stephan R. Vavricka

Division of Gastroenterology and Hepatology; University Hospital, Raemistrasse 100, CH-8091 Zurich, Switzerland

Correspondence to:

Stephan R. Vavricka, MD

Division of Gastroenterology and Hepatology

University Hospital

Raemistrasse 100

CH-8091 Zurich

Switzerland

Tel: $\quad 0041442552124$

Fax: 0041442554503

Email: stephan.vavricka@usz.ch 
Letter

To the Editor:

We read with great interest the article by Chen and colleagues regarding prophylactic lamivudine use in chemotherapy-associated hepatitis B reactivation in non-Hodgkin's lymphoma. ${ }^{1}$ Reactivation of hepatitis B virus (HBV) is a well-recognized complication in patients with chronic HBV infection who receive immunosuppressive or cytotoxic therapy. ${ }^{2}$ Many recent studies suggest a clear benefit of lamivudine in terms of clinical and virological HBV reactivation, overall mortality, HBV-related mortality and interruptions or discontinuations in the immunosuppressive treatment. ${ }^{3}$ Furthermore, various different chemotherapeutic agents such as corticosteroids and anthracyclines are proven risk factors for HBV reactivation. ${ }^{4}$ We would like to emphasize to consider a HBV reactivation after discontinuation of a prednisone therapy and report a patient with HBV-infection who developed HBV reactivation following corticoid treatment of ulcerative colitis.

A 43-year-old male patient was diagnosed with ulcerative colitis in 2003. Initially, the patient responded well to a systemic therapy with mesalazine 3g/d. In May 2005 he developed another episode of ulcerative colitis, this time more severe with 4 to 6 bowel movements per day and intermittent rectal bleeding, mild fever $\left(38.8^{\circ} \mathrm{C}\right)$, oligoarticular arthritis and lower abdominal tenderness. A colonoscopy was performed, which showed a left-sided colitis. A therapy with prednisone $50 \mathrm{mg} / \mathrm{d}$ and azathioprine $50 \mathrm{mg} / \mathrm{d}$ was initiated. Of note, LFTs were normal at this time. Two weeks later, the patient was well, prednisone was completely tapered and azathioprine was increased to $100 \mathrm{mg} / \mathrm{d}$. Three months later the patient presented to the emergency department in an outside hospital with nausea, vomiting, right upper quadrant pain, and jaundice. LFTs were markedly elevated. AST was 2193 U/l, ALT 3396 U/L, GGT 69 U/L, bilirubine $18.25 \mathrm{mg} / \mathrm{dl}$, prothrombine time $31 \%$, and C-reactive protein was $4 \mathrm{mg} / \mathrm{l}$. Further work-up revealed highly replicative hepatitis B (HBV-DNS PCR $>110^{\prime} 000^{\prime} 000 \mathrm{IE} / \mathrm{ml}$ ) reactivation (HBs-Ag pos, antiHbclgM neg, HBe-Ag neg, anti-HBe pos). Other viral serologies for hepatitis A, C, D, and HIV were all negative. Therapy with azathioprine was stopped. Abdominal sonography revealed ascites, normal calibre intra- and extrahepatic bile ducts and no gallstones. During the hospitalisation stool frequency increased to 6 per day with bloody diarrhea. The patient was started on prednisone $40 \mathrm{mg} / \mathrm{d}$, which had to be increased to $50 \mathrm{mg} / \mathrm{d}$ after 10 days. Colonoscopy revealed a mild flare of ulcerative colitis. In the course LFT's decreased (AST $213 \mathrm{U} / \mathrm{l}$, ALT $364 \mathrm{U} /$ ) and the patient could be discharged on $50 \mathrm{mg}$ prednisone/d. 
One week later the patient presented with abdominal pain, fatigue, progressive jaundice and extension of the abdomen. On admission the patient showed signs of hepatic failure with elevated LFT's and ascites. Paracentesis showed spontaneous bacterial peritonitis. The patient was transferred to our tertiary care center due to severe liver failure with hepatic encephalopathy (Grade 3) and coagulopathy (Factor $\vee 7 \%$, PT 10\%, aPTT $88 \mathrm{sec})$. We immediately started a therapy with lamivudine and listed the patient for superurgent liver transplantation, which could be performed 24hours latter. One day after transplantation the patient presented with primary graft non function, which required immediate re-transplantation. After re-transplantation the patient recovered quickly. To prevent HBV re-infection, we initiated a long-term prophylaxis with anti-HBs immunoglobulins in combination with lamivudine. Three and a half years after re-transplantation there is no evidence for HBV reinfection and the patients has fully recovered. This case highlights the importance of HBV screening in IBD patients requiring an immunosuppressive therapy such as corticosteroids, methotrexate, azathioprine or new biological agents such as anti-TNF agents. ${ }^{5,6}$ In our opinion, HBV carriers with IBD requiring such a treatment should also be treated prophylactically with a nucleoside or nucleotide analogues approved for the treatment of HBV infection.

\section{References}

1. Hsu C, Hsiung CA, Su IJ, Hwang WS, Wang MC, Lin SF, Lin TH, Hsiao HH, Young JH, Chang MC, Liao YM, Li CC, Wu HB, Tien HF, Chao TY, Liu TW, Cheng AL, Chen PJ. A revisit of prophylactic lamivudine for chemotherapy-associated hepatitis B reactivation in non-Hodgkin's lymphoma: a randomized trial; Hepatology. 2008;47:844-53.

2. Perrillo RP. Acute flares in chronic hepatitis $B$ : the natural and unnatural history of an immunologically mediated liver disease. Gastroenterology. 2001 Mar;120(4):1009-22.

3. Katz LH, Fraser A, Gafter-Gvili A, Leibovici L, Tur-Kaspa R. Lamivudine prevents reactivation of hepatitis B and reduces mortality in immunosuppressed patients: systematic review and metaanalysis J Viral Hepat. 2008;15:89-102.

4. Lalazar G, Rund D, Shouval D. Screening, prevention and treatment of viral hepatitis B reactivation in patients with haematological malignancies. Br J Haematol. 2007;136:699-712. 
5. Chang JT, Lichtenstein GR. Drug insight: antagonists of tumor-necrosis factor-alpha in the treatment of inflammatory bowel disease. Nat Clin Pract Gastroenterol Hepatol. 2006;3:220-8.

6. Lubel JS, Testro AG, Angus PW. Hepatitis B virus reactivation following immunosuppressive therapy: guidelines for prevention and management. Intern Med J. 2007;37:705-12.

\section{Acknowledgements}

This study was supported by a research grant from the Swiss National Science Foundation grant 320000114009/1 (to SRV), 3347CO-108792 (Swiss IBD Cohort) and a grant of the Zurich Center of Integrative Human Physiology. 